\title{
EL TRABAJO GRUPAL DEL ESTUDIANTE EN FORMATO CONGRESO PROFESIONAL CON EVALUACIÓN FORMATIVA Y TRIÁDICA EN EL MÁSTER DE PROFESORADO DE EDUCACIÓN SECUNDARIA
}

The group student work format Professional Congress with formativen assessment and triaddic in the Unviersity Máster in Secondary

O trabalho autónomo do estudante em formato congresso profissional com avaliação formativa e triádica no mestrado de formação de professores no ensino secundário

\section{Nuria Ureña Ortín (1)}

Pedro A. López Miñarro (2)

Este estudio se ha llevado a cabo dentro del Grupo Innovación Docente metodologías activas, TICS y evaluación en el área de Didáctica de la Expresión Corporal y Musical (Grupo MATE)

(1) Universidad de Murcia, España. Teléfono: +34 86888 8470. Correo electrónico: nuriaur@um.es

(2) Universidad de Murcia, España. Teléfono: +34 86888 7051. Correo electrónico: palopez@um.es

\section{Resumen}

En esta experiencia se presenta una metodología innovadora e interdisciplinar para la elaboración del trabajo grupal autónomo del estudiante en el ámbito universitario. Para ello, los docentes de dos asignaturas del Máster Universitario de Formación de Profesorado en Educación Secundaria Obligatoria y Bachillerato organizaron la realización del trabajo grupal autónomo del estudiante a través de un formato de Congresos Profesionales. Además, esta experiencia se desarrolló bajo un proceso de evaluación formativa y se implementó un proceso de evaluación tríadica en las presentaciones orales, utilizando como instrumento una escala de valoración. Los resultados mostraron una satisfacción muy positiva por parte del estudiante, así como un alto grado en la adquisición de las competencias, tanto generales como específicas. Por tanto, se establece como necesario la introducción de este tipo de metodologías activas, así como la incorporación del alumnado en los procesos de evaluación en las enseñanzas universitarias.

Palabras clave: Triádica; congreso; autónomo; rúbrica de puntuación; autoevaluación; coevaluación 


\begin{abstract}
In this experience, an innovative and interdisciplinary methodology for the development of autonomous group work of the student in the university environment is presented. For this, the teachers of two subjects of the Master's Degree in Teacher Training in Compulsory Secondary Education and Baccalaureate organized the autonomous group work of the student through a format of Professional Congresses. In addition, this experience was developed under a formative assessment process and a triadic evaluation process was implemented in the oral presentations, using as an instrument a rating scale. The results showed a very positive satisfaction on the part of the student, as well as a high degree in the acquisition of the competences, both general and specific. Therefore, it is established as necessary the introduction of this type of active methodologies, as well as the incorporation of students in the evaluation processes in university education.
\end{abstract} Keywords: Triadic; congress; autonomous; scoring rubric; self-assessment; coevaluation

\title{
Resumo
}

Nessa experiência apresenta-se uma metodologia inovadora e interdisciplinar para o desenvolvimento do trabalho de grupo autónomo do aluno do ensino universitário. Para o efeito, os professores de duas disciplinas do Mestrado em Formação de Professores no Ensino Secundário organizaram a realização de trabalho de grupo autónomo por parte dos alunos, através de um formato de Congressos Profissionais. Além disso, esta experiência desenvolveu-se, segundo um processo de avaliação formativa e um processo de avaliação triádica nas apresentações orais, utilizando como instrumento uma escala de valoração. Os resultados mostraram uma satisfação muito positiva por parte dos alunos, bem como um alto grau de aquisição das competências gerais e específicas. Nesse sentido, estabelece-se como necessário a introdução deste tipo de metodologias ativas, bem como a incorporação dos estudantes nos processos de avaliação no ensino universitário.

Palavras-chave: Triádica; congresso; autónomo; rubrica de pontuação; autoavaliação e coavaliação 


\section{Introducción}

Los Másteres universitarios tienen como finalidad la adquisición por el estudiante de una formación avanzada, de carácter multidisciplinar, orientada a la especialización académica o profesional, o bien a promover la iniciación en tareas investigadoras. En el caso del Máster Universitario en Formación del Profesorado de Educación Secundaria, marco en el que se desarrollada esta experiencia, se hace hincapié en la primera vertiente (Ureña \& Ruiz, 2012). A este propósito hay que añadir el paradigma competencial, donde el estudiante debe de edificar su conocimiento y lograr una formación profesional y personal más completa (Fernández-Jiménez, Polo \& Fernández-Cabeza, 2017).

En este marco competencial y multidisciplinar, se propone una metodología basada en la innovación y el trabajo interdisciplinar en formato Congreso Profesional o Académico (Civila, Fernández \& Guerreo, 2010) para elaborar el trabajo grupal autónomo. Autores como Pérez y Antolín (2016) concretan que es necesario nuevas fórmulas docentes para lograr sacar al alumnado de la "comodidad" en la que se encuentra. Para conseguir un resultado eficaz en este proceso es necesario complementar este trabajo con un procedimiento de tutorización y seguimiento del proceso de aprendizaje del alumnado por parte del docente (Pérez- Pueyo, Casado \& Hortigüela, 2019). En este sentido, Manrique (2017, p. 36) especifica que la autonomía no se alcanza con el simple "dejar hacer" cuando se encarga un trabajo en grupo autónomo. Para ayudar a desarrollar la autonomía en los alumnos es fundamental acompañarlos en el recorrido y ayudarles a que tomen conciencia de los errores y aciertos que realizan.

Por tanto, se planteó una nueva fórmula docente cuyo formato fue el de los Congresos Profesionales y donde fueron los propios estudiantes los protagonistas y agentes activos de todo el proceso con la guía de los docentes que actuaron como comité organizador y evaluador de este.

\section{Contextualización}

La experiencia de aplicación, a través de un Congreso Profesional, se puso en práctica en las asignaturas que se indican en la Figura 1 relacionándolas con las competencias profesionales que deben adquirir los estudiantes. 


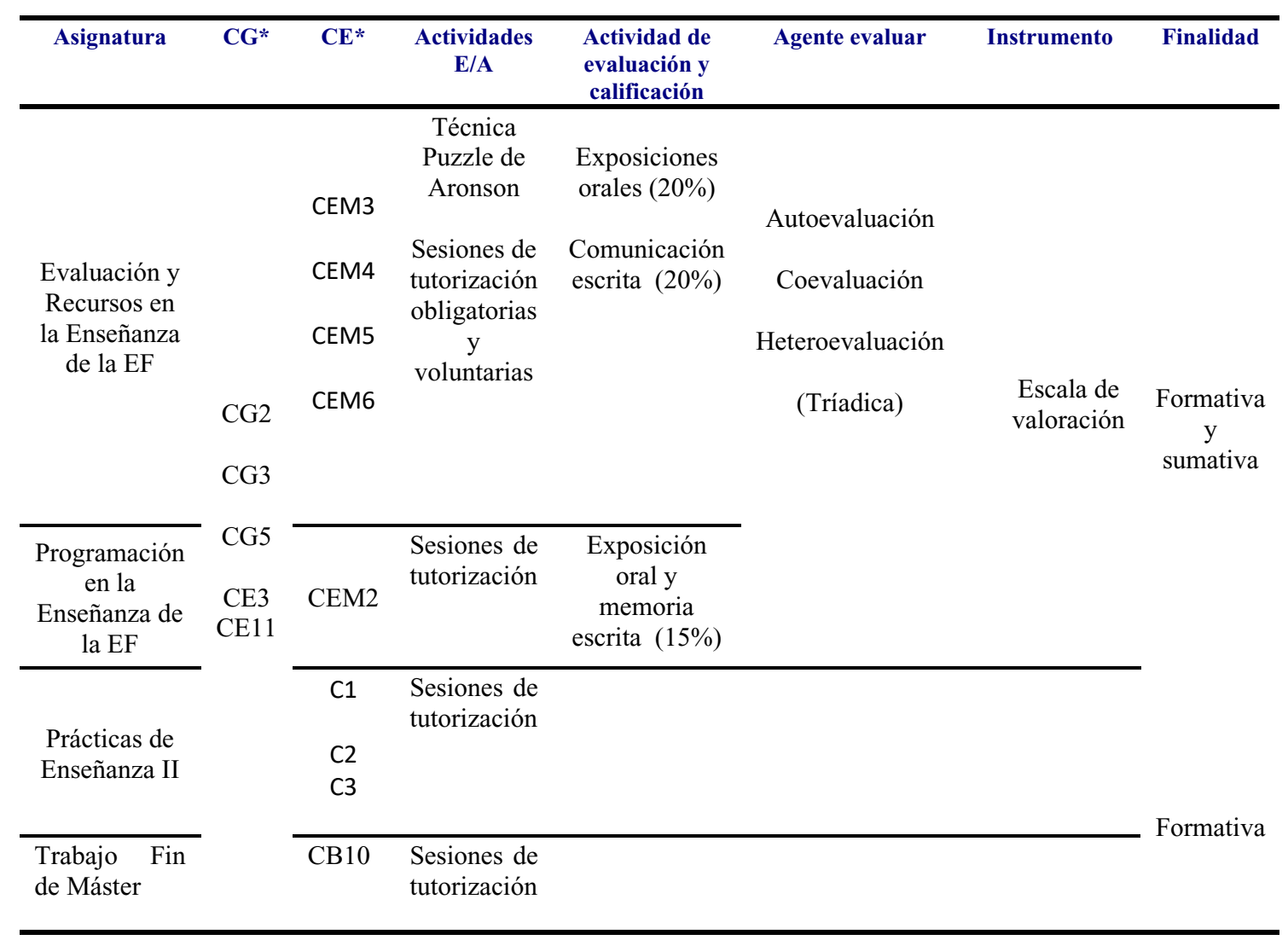

Figura 1. Relación entre asignaturas, competencias, actividades de E/A y evaluación.

\section{Diseño y desarrollo}

Para llevar a cabo esta experiencia se valoró la posibilidad de modificar el formato de elaboración del trabajo grupal autónomo desde las siguientes líneas de actuación: (a) una metodología innovadora de carácter interdisciplinar en formato Congreso Profesional (b) que la propuesta adquiriese un planteamiento de evaluación de carácter formativo (Pérez-Pueyo et al. 2019); y (c) que involucrase al alumnado en el propio proceso de aprendizaje y el de sus compañeros (evaluación triádica) (Pérez-Pueyo, 2016; Pérez-Pueyo \& Sobejano, 2017).

El primer día de clase se planteó a los estudiantes la posibilidad de utilizar esta metodología para la adquisición de competencias, tanto genéricas como específicas, de diversas asignaturas (Figura 2). 


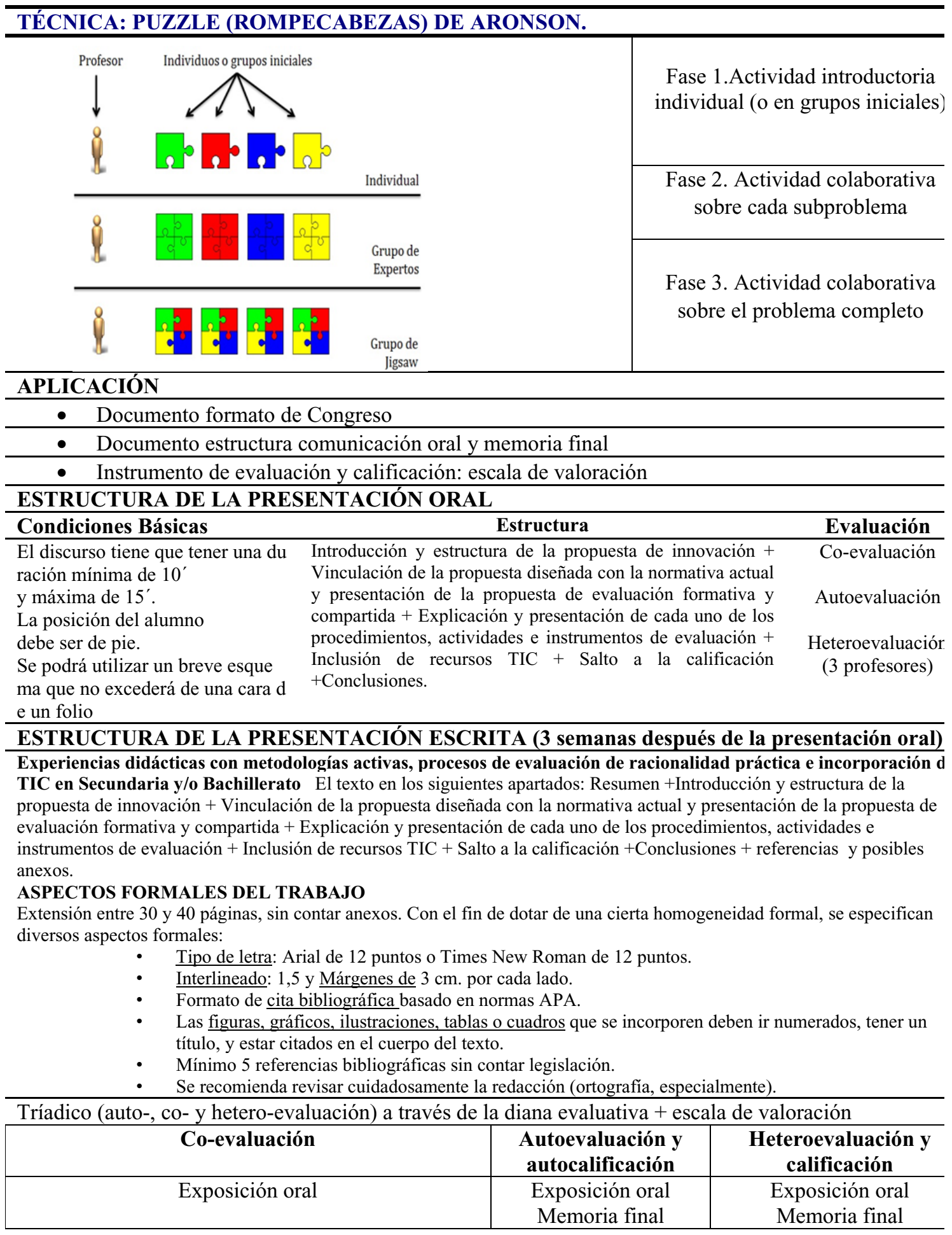

Figura 2. Actividades realizadas para desarrollo trabajo grupal autónomo.

La propuesta fue explicada en clase, tutorizada y posteriormente expuesta en un único día (3 horas) para asistir y participación en un Congreso (Figura 3). En este sentido, se 
intentó concienciar al alumnado de la necesidad de asistir y participar en congresos ya que son una fuente de conocimiento y aprendizaje para todo profesional, se comparten experiencias con otros profesionales y potencian las habilidades, tanto sociales como comunicativas.

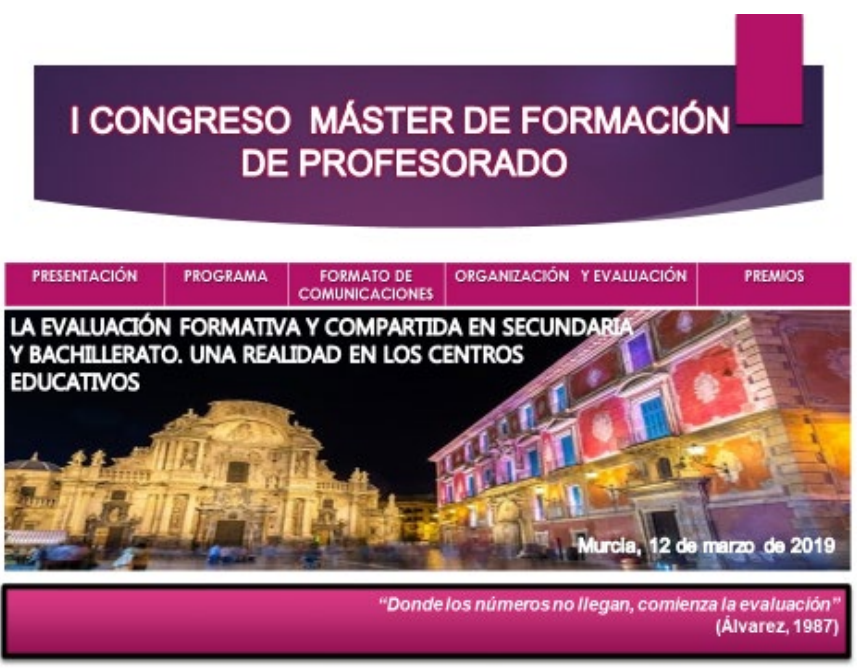

Figura 3. I Congreso Máster de Formación de profesorado y publicación "Call for Papers”.

\subsection{Fases para la puesta en práctica del Congreso}

1. Explicación del formato del Congreso para exposiciones orales, comunicación escrita y el instrumento de evaluación que se iba a utilizar (evaluación triádica). Se utilizó una sesión a través la metodología aprendizaje cooperativo con la técnica del puzzle de Aronson (Figura 2). Los instrumentos de evaluación para la evaluación tríadica fueron una rúbrica para la co-evaluación y autoevaluación con diana gráfica y una escala de valoración para la memoria escrita donde los tres agentes implicados utilizaban los mismos instrumentos (López Pastor y Pérez-Pueyo, 2017; Pérez-Pueyo y Sobejano, 2017).

2. Procesos de evaluación formativa a partir de la tutorización y orientación efectiva, utilizando feedback (Pérez-Pueyo et al., 2019). Las tutorías, gestionadas por el comité organizador y evaluador del Congreso, fueron decisivas en este proceso de aprendizaje (Figura 4).

3. Inclusión de premios para motivar al alumnado. Para incrementar, tanto la motivación de los estudiantes como su implicación en el Congreso (Figura 5), se adoptaron algunas estrategias propias de las mecánicas de juego. 
TUTORIZACIÓN: para una correcta búsqueda de contenidos, selección de información y la maduración de los formatos que conformaron las presentaciones orales y la presentación final de la comunicación

$1^{\text {a }}$ Fase $\quad 2^{\text {a }}$ Fase

Enviar un resumen ampliado (máximo 2páginas) describiendo el contexto de la innovación, curso y contenido; este resumen era revisado por los docentes (comité organizador $\mathrm{CO}$ ) y podía ser aceptado o rechazado. En caso de rechazo, el CO explicaba, a través de la tutoría, las razones del Los alumnos enviaron el trabajo completo según la normativa establecida en el Congreso.

Posteriormente, el alumno recibía la valoración y posibles sugerencias de mejora a incorporar en la versión final. rechazo y el grupo debía, revisarlo y enviarlo de nuevo

\begin{tabular}{ccc}
\hline & Feedback: agente y momento & \\
\hline Resumen ampliado & $\begin{array}{c}\text { Presentación oral } \\
\text { (exposiciones) }\end{array}$ & $\begin{array}{c}\text { Presentación trabajo completo } \\
\text { escrito }\end{array}$ \\
\hline $\begin{array}{c}\text { Comité organizador } \\
\text { (docentes) }\end{array}$ & $\begin{array}{c}\text { Comité organizador } \\
\text { (docentes) } \\
\text { El resto de los grupos }\end{array}$ & $\begin{array}{c}\text { Comité organizador } \\
\text { (docentes) }\end{array}$ \\
\hline
\end{tabular}

Figura 4. Fases en la entrega de las presentaciones y trabajo completo y proceso de revisiones.

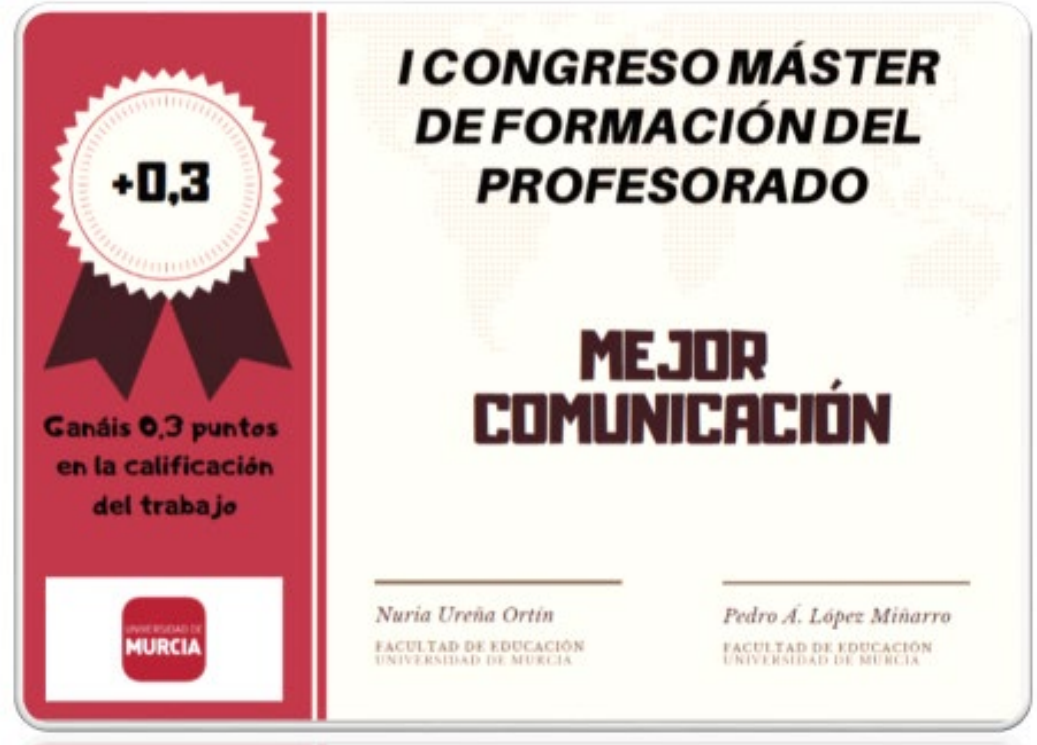

Figura 5. Premio a la mejor comunicación.

Esta experiencia de buena práctica será evaluada por dos instrumentos estandarizados que ha generado la Red de Evaluación Formativa:

a) La Escala para la autopercepción de competencias de los estudiantes (Salcines, González, Ramírez \& Martínez, 2018).

b) La Escala de percepción sobre Metodologías EMPEF al finalizar la asignatura (Castejón, Santos \& Palacios 2015). 


\section{Evaluación y conclusiones}

Nuestra experiencia, todavía sin finalizar, revela que tras los resultados obtenidos en el ítem 8 de la escala de percepción sobre metodologías EMPEF el estudiante mostró una satisfacción global positiva (30,43\% muy satisfecho y 60,86\% bastante satisfecho), así como permitió la adquisición de competencias (ítem 3$)$ profesionales $(26,08 \%$ mucho y $65,21 \%$ bastante). De igual forma se obtuvieron beneficios en competencias genéricas como la capacidad de expresión oral, tan importante y transversal para un futuro docente (Pérez-Pueyo, 2015). Desde el punto de vista de la evaluación formativa tríadica permitió obtener una mayor calidad en el documento escrito identificando con más claridad los aspectos a valorar y los niveles de logro. Además, se recibió información desde tres agentes (Pérez-Pueyo, 2016).

Las líneas de mejora se orientarán a ajustar el número de horas dedicado por los grupos (carga de trabajo y falta de coordinación con profesores de las diferentes asignaturas). También se procurará anticipar los plazos de tutorización para que haya más posibilidad de retroalimentación. Finalmente, y para completar todas las fases de un Congreso Profesional, se elaborarán certificados de participación y exposición a todos los estudiantes, se incluirán otras actividades el día de las exposiciones (pausa para el café, los descansos y la clausura del Congreso).

\section{Referencias}

Castejón, F.J., Santos, M.L. \& Palacios, A. (2015). Cuestionario sobre metodología y evaluación en formación inicial en educación física. Revista Internacional de Medicina y Ciencias de la Actividad Física y el Deporte, 58, 245-267. Recuperado de http://cdeporte.rediris.es/revista/revista58/artescala566.htm

Civila, A, Fernández, A. J. \& Guerreo, P. (2010). Aprendizaje holístico, autónomo y colaborativo mediante un congreso de alumnos En C. Ruiz. (Coord.), Innovación educativa y nuevos modelos de enseñanza-aprendizaje en el contexto universitario (pp. 53-98). Málaga, Servicio de Publicaciones de la Universidad de Málaga.

Fernández- Jiménez, C., Polo, Mª T. \& Fernández Cabeza, M. a (2017). Aplicación de la autoevaluación en una experiencia de Aprendizaje Basado en Problemas con alumnado de educación en asignaturas relacionadas con la discapacidad. Estudios Sobre Educación, 32, 73-93. 
López-Pastor, V. M. \& Pérez-Pueyo, A. (Coords.) (2017). Evaluación formativa y compartida en educación: experiencias de éxito en todas las etapas educativas. León: Universidad de León. Recuperado de https://buleria.unileon.es/handle/10612/599

Manrique, J. C (2017). El potencial de los proyectos de aprendizaje tutorados en la formación inicial del profesorado. Revista Infancia, Educación y Aprendizaje, $3(2), 36-41$.

Pérez, C. \& Antolí, A. (2016). Desarrollar competencias en la educación superior con trabajo autónomo y de investigación. Opción (32), 12, 238-253. Recuperado de http://www.redalyc.org/pdf/310/31048903012.pdf

Pérez-Pueyo, A. (2015). Escala de valoración para la evaluación tríadica de exposiciones orales. En N. González, I. Salcines y M. ${ }^{\text {a } ~ T . ~ G a r c i ́ a, ~ T e n d e n c i a s ~}$ emergentes en evaluación formativa y compartida en docencia. El papel de las nuevas tecnologías II. (pp. 1257-1288). Santander: Universidad de Cantabria.

Pérez-Pueyo, A. (2016). El estilo actitudinal en educación física: evolución en los últimos 20 años. Retos: nuevas tendencias en educación física, deporte y recreación, (29), 207-215. Recuperado de https://recyt.fecyt.es/index.php/retos/article/view/38720

Pérez-Pueyo, Á., \& Sobejano, M. (2017). Elaboración de instrumentos (escalas de valoración y graduadas) para la evaluación formativa. Revista Infancia, Educación y Aprendizaje, 3(2), 808-814. Recuperado de https://revistas.uv.cl/index.php/IEYA/article/view/823

Pérez-Pueyo, A., Casado, O., \& Hortigüela, D. (2019). La evaluación formativa, la autorregulación y la secuenciación de competencias. En J. Manso y J. Moya, Profesión y profesionalidad docente. Una acción educativa comprometida con el desarrollo humano (pp. 103-120). Andalucía: ANELE.

Salcines, I., González-Fernández, N., Ramírez-García, A. \& Martínez-Mínguez, L. (2018). Validación de la Escala de Autopercepción de Competencias Transversales y Profesionales de Estudiantes de Educación Superior. Profesorado. Revista de Currículum y Formación de Profesorado, 22(3), 31-51. Recuperado de http://revistaseug.ugr.es/index.php/profesorado/article/view/7989 
Ureña, N. \& Ruiz, R. (2012). Experiencia de evaluación formativa y compartida en el Máster Universitario en Formación del Profesorado de Educación Secundaria. Psychology, Society, \& Education, 4(1), 29-44. Recuperado de http://ojs.ual.es/ojs/index.php/psye/article/view/552 